\title{
EXISTENCE OF SOLUTIONS OF IMPLICIT DIFFERENTIAL INCLUSIONS
}

\author{
A. ANGURAJ* AND K. BALACHANDRAN
}

\begin{abstract}
We prove the existence of solutions for the implicit differential inclusions of the form

$$
\begin{aligned}
& \dot{x}(t) \in A(t, x)+F(t, x, \dot{x}) \text { for } t \in I, \quad \text { a.e. } \\
& x(r)=0
\end{aligned}
$$
\end{abstract}

with $I=[r, r+a]$ by using the Covitz-Nadler fixed point theorem.

\section{Introduction}

The purpose of this paper is to prove the existence of solutions of implicit $\delta$ differential inclusions $\dot{x}(t) \in A(t, x)+F(t, x, \dot{x})$ where $F$ is a setvalued function, Lipschitz continuous with respect to its last two variables and $A$ is a single valued continuous function satisfying the Lipschitz condition. In [1] we assume the existence of the classical solutions of the above problem and proved that the solution set is a retract of $C(I, E)$ where $E$ is a Banach space. In this paper we prove an existence theorem by using the Covitz-Nadler fixed point theorem.

Fixed point theorems are used as a tool to prove the existence of solutions of differential inclusions [2]. Papageorgiou [7] used the Schauder-Tichonoff fixed point theorem to prove the existence of solutions of the above problem with

Received November 20, 1991; revised June 20, 1992. 
delay and without the term $A(t, x)$. Ricceri [8] and Kisielewicz [5] considered the above equation without the term $A(t, x)$ where as the former studied the classical solution sets with some applications while the latter proved the existence of solutions by using the Covitz-Nadler fixed point theorem.

\section{Preliminaries}

Let $I$ be a compact interval of the real line and let $F: I \rightarrow 2^{R^{n}}$ be a setvalued function. Denote by $S(F)$ the collection of all $L$-integrable (Lebesgue integrable) functions $u: I \rightarrow R^{n}$ having the property that $u(t) \in F(t)$ a.e. in $I$. The set $S(F)$ is said to be the trajectory integrals of the setvalued function $F$. Especially interesting, from the point of view of functional differential inclusions, are subtrajectory integrals of such family of setvalued functions $F(z): I \rightarrow 2^{R^{n}}$ depending on a parameter $z$. The subtrajectory integrals of such family setvalued functions will be denoted by $S(F)(z)$.

Let $L\left(I, R^{n}\right)$ be the space of all $L$-integrable functions $u: I \rightarrow \mathbb{R}^{n}$. A setvalued map $F: I \rightarrow \operatorname{Comp}\left(\mathbb{R}^{n}\right)$ (non empty compact subsets of $\mathbb{R}^{n}$ ) is said to be integrably bounded if there exists a non negative function $u \in L\left(I, R^{n}\right)$ such that $F(t) \subset u(t) B$ a.e. in $I$, where $B$ is a closed unit ball in $R^{n}$. Let $\left(\mathcal{A}\left(I, R^{n}\right), d\right)$ be the metric space of all setvalued functions $F: I \rightarrow \operatorname{Comp}\left(R^{n}\right)$ that are $L$ measurable (Lebesgue) and integrably bounded with a metric $d$ defined by

$$
d(F, G)=\int_{I} H(F(t), G(t)) \text { for } F, G \in \mathcal{A}\left(I, R^{n}\right) .
$$

where the Hausdorff metric $H$ is defined by

$$
\begin{aligned}
& H(A, B)=\max \{\bar{H}(A, B), \bar{H}(B, A)\} \text { where } \\
& \bar{H}(B, A)=\max _{b \in B}\{\operatorname{dist}(b, A), \operatorname{dist}(b, A)\}=\inf _{a \in A}|b-a|
\end{aligned}
$$

We shall consider a family of all setvalued functions $F: I \times C\left(I, R^{n}\right) \times$ $L\left(I, R^{n}\right) \rightarrow \operatorname{Comp}\left(\mathbb{R}^{n}\right)$ such that $F(\cdot, x, z) \in \mathcal{A}\left(I, R^{n}\right)$ for each fixed $(x, z) \in$ $C\left(I, R^{n}\right) \times L\left(I, R^{n}\right)$. 
We say that the setvalued map $F$ is Lipschitz continuous with respect to its last two variables if there is an $L$-integrable function $k: I \rightarrow R$ such that for every $x, \bar{x} \in C\left(I, R^{n}\right), z, \bar{z} \in L\left(I, R^{n}\right)$ and a.e. $t \in I$ one has

$$
H\left(F(t, x, z), F(t, \bar{x}, \bar{z}) \leq k(t) \max \left(|x-\bar{x}|_{t},|z-\bar{z}|_{t}\right)\right.
$$

where

$$
\begin{aligned}
|x-\bar{x}|_{t} & =\sup _{r \leq s \leq t}|x(s)-\bar{x}(s)| \text { and } \\
|z-z|_{t} & =\int_{r}^{t}|z(s)-\bar{z}(s)| d s .
\end{aligned}
$$

We also use the norm \|\|$_{0}$ as $\|G(t)\|_{0}=H(G(t),\{0\})$.

We prove our existence theorem by using the following fixed point theorem.

Theorem (Covitz-Nadler). Let $(X, \rho)$ be a complete metric space and $G: X \rightarrow c l(X),($ Closer of $X)$, a setvalued contraction mapping, that is, such that $H(G(x), G(y)) \leq k \rho(x, y)$ for every $x, y \in X$ with any $k \in[0,1)$. Then there exists $x \in X$ such that $x \in G(x)$.

The existence of subtrajectory integrals in this paper are due to the continuous selection theorems of Fryszkowski [4] and Michael [6].

\section{Existence}

In this section we prove the existence of solutions of implicit differential inclusions of $\delta$ the form

$$
\begin{aligned}
& \dot{x}(t) \in A(t, x)+F(t, x, \dot{x}) \text { for } t \in I, \text { a.e. } \\
& x(r)=0
\end{aligned}
$$

with $I=[r, r+a]$. We assume the following hypotheses.

(i) $F: I \times C\left(I, R^{n}\right) \times L\left(I, R^{n}\right) \rightarrow \operatorname{Comp}\left(R^{n}\right)$ is such that $F(\cdot, x, z) \in$ $\mathcal{A}\left(I, R^{n}\right)$ for fixed $(x, z)$ and $F$ is Lipschitz continuous on $C\left(I, R^{n}\right) \times$ $L\left(I, R^{n}\right)$ with respect to its last two variables. 
(ii) $A: I \times C\left(I, R^{n}\right) \rightarrow R^{n}$ is single valued continuous function such that $|A(t, x)-A(t, y)| \leq k(t)|x-y|_{t}$ where $k: I \rightarrow R$ is a Lebesgue integrable function.

Let $T: L\left(I, R^{n}\right) \rightarrow C\left(I, R^{n}\right)$ be defined by

$$
\left(T^{\prime} u\right)(t)=\int_{r}^{t} u(s) d s, \text { for } u \in L\left(I, R^{n}\right), t \in I .
$$

Theorem. Under the assumptions (i) and (ii) the initial value problem (1) has at least one solution.

Proof. Let $F \circ T$ be defined on $I \times L\left(I, R^{n}\right)$ by setting $(F \circ T)(t, z)=$ $F(t, T z, z)$ for $(t, z) \in I \times L\left(I, R^{n}\right)$ and let $S(F \circ T)(z)$ the subtrajectory integrals of $(F \circ T)(\cdot, z)$ for each fixed $z \in L\left(I, R^{n}\right)$.

Now define the setvalued mapping $G(t, z)$ on $I \times L\left(I, R^{n}\right)$ to the compact subsets of $R^{n}$ as

$$
G(t, z)=A(t, T z)+(F \circ T)(t, z)
$$

Let

$$
S(G)(z)=\left\{w \in L\left(I, R^{n}\right): w(t) \in A(t, T z)+S(F \circ T)(z)\right\}
$$

be the subtrajectory integrals of $G(\cdot, z)$ for each fixed $z \in L\left(I, R^{n}\right)$. Clearly $G \in \mathcal{A}\left(I, R^{n}\right)$ and $S(G)$ is a nonempty closed and bounded subset of

$$
L_{G}=\left\{w \in L\left(I, R^{n}\right):|w(t)| \leq\|G(t, z)\|_{0} \text { for a.e. } t \in I\right\}
$$

Let $u$ be an arbitrary element of $S(F \circ T)(z)$. By Fillipov's theorem [3] there is a $v \in S(F \circ T)(\bar{z})$ such that

$$
\begin{aligned}
|u(t)-v(t)| & <\operatorname{dist}(u(t), F(t, T \bar{z}, \bar{z})) \\
& \leq H(F(t, T z, z), F(t, T \bar{z}, \bar{z}) \\
& \leq k(t) \max \left(|T z-T \bar{z}|_{t},|z-\bar{z}|_{t}\right)
\end{aligned}
$$

for $t \in I$ a.e. 
Let $m=3 / M, M \in(0,1)$ and let us define the norm on $L\left(I, R^{n}\right)$ as

$$
\begin{aligned}
\|\omega\| & =\int_{r}^{r+a} e^{-m k(t)}|\omega(t)| d t \quad \text { for } \omega \in L\left(I, R^{n}\right) \text { with } \\
K(t) & =\int_{r}^{t} k(s) d s
\end{aligned}
$$

Now

$$
\begin{aligned}
\bar{H}(S(G)(z), S(G)(\bar{z})) & =\bar{H}(A(t, T z)+S(F \circ T)(z), A(t, T \bar{z})+S(F \circ T)(\bar{z})) \\
& \leq\|A(t, T z)-A(t, T \bar{z})\|+\|u-v\|
\end{aligned}
$$

for each $u \in S(F \circ T)(z)$ and any $v \in S(F \circ T)(\bar{z})$.

Now,

$$
\begin{aligned}
\|u-v\|= & \int_{r}^{r+a} e^{-m K(t)}|u(t)-v(t)| d t \\
\leq & \int_{r}^{r+a} k(t) e^{-m K(t)} \max \left(|T z-T \bar{z}|_{t},|z-\bar{z}|_{t}\right) d t \\
\leq & \int_{r}^{r+a} k(t) e^{-m K(t)} \sup _{r \leq s \leq t}|T z(s)-T \bar{z}(s)| d t \\
& +\int_{r}^{r+a} k(t) e^{-m K(t)}\left(\int_{r}^{t}|z(s)-\bar{z}(s)| d s\right) d t
\end{aligned}
$$

But,

$$
\begin{aligned}
& \int_{r}^{r+a} k(t) e^{-m K(t)} \sup _{r \leq s \leq t}|T z(s)-T \bar{z}(s)| d t \\
\leq & \int_{r}^{r+a} k(t) e^{-m K(t)}\left(\int_{r}^{t}|z(s)-\bar{z}(s)| d s\right) d t \\
= & -(1 / m) e^{-m K(r+a)} \int_{r}^{r+a}|z(s)-\bar{z}(s)| d s \\
& +1 / m \int_{r}^{r+a} e^{-m K(t)}|z(t)-\bar{z}(t)| d t \\
\leq & \frac{M}{3}\|z-\bar{z}\|
\end{aligned}
$$

and

$$
\int_{r}^{r+a} k(t) e^{-m K(t)}\left(\int_{r}^{t}|z(s)-\bar{z}(s)| d s\right) d t \leq \frac{M}{3}\|z-\bar{z}\|
$$


Similarly,

$$
\begin{aligned}
\| A(t, T z) & -A(t, T \bar{z}) \|=\int_{r}^{r+a} e^{-m K(t)}|A(t, T z)-A(t, T \bar{z})| d t \\
\leq & \int_{r}^{r+a} k(t) e^{-m K(t)}|T z-T \bar{z}|_{t} d t \\
\leq & \int_{r}^{r+a} k(t) e^{-m K(t)} \sup _{r \leq s \leq t}|T z(s)-T \bar{z}(s)| d t \\
\leq & \frac{M}{3}\|z-\bar{z}\| .
\end{aligned}
$$

Therefore,

$$
\bar{H}(S(G)(z), S(G)(\bar{z})) \leq M\|z-\bar{z}\|
$$

In a similar way we obtain

$$
\bar{H}(S(G)(\bar{z}), S(G)(z)) \leq M\|z-\bar{z}\|
$$

Hence $H(S(G)(z), S(G)(\bar{z})) \leq M\|z-\bar{z}\|$ for each $z, \bar{z} \in L\left(I, R^{n}\right)$.

Put $X=L\left(I, R^{n}\right)$ and $\rho\left(z_{1}, z_{2}\right)=\left\|z_{1}-z_{2}\right\|$ for every $z_{1}, z_{2} \in X$. Obviously $(X, \rho)$ is a complete metric space. Since $M \in(0,1)$ and $S(G)(z) \in \operatorname{cl}\left(L\left(I, R^{n}\right)\right)$ for every $z \in X, S(G)$ is setvalued contractive mapping of $X$ into $\operatorname{cl}(X)$. Now by Covitz-Nadler fixed point theorem there is $z \in X$ such that $z \in S(G)(z)$. Put $x=T z$. Then we obtain $x(r)=0$ and $\dot{x}(t) \in A(t, x)+F(t, x, \dot{x})$ for a.e. $t \in I$. Therefore $x$ is a solution of (1).

\section{References}

[1] A. Anguraj and K. Balachandran, "On the solution sets of differential inclusion in Banach spaces", Tamkang J. Math. 23 (1992), No. 2, 57-63.

[2] J. P. Aubin and A. Cellina, "Differential Inclusions", Springer-Verlag, Berlin, 1984.

[3] J. R. Bridgland, T. F., "Trajectory integrals of setvalued functions", Pacific J. Math., 33 (1970), 43-67.

[4] A. Fryszkowski, "Continuous selections for a class of nonconvex multivalued maps", Studia Math., 76 (1983), 163-174.

[5] M. Kisielewicz, "Subtrajectory integrals of setvalued functions and neutral functional differential inclusions", Funkcialaj Ekvacioj, 32 (1989), 163-189.

[6] E. Michael, "Continuous selections I", Ann. Math., 63 (1956), 361-382. 
[7] N. S. Papageorgiou, "On the theory of functional differential inclusions of neutral type in Banach spaces", Funkcialaj Ekvacioj, 31 (1988), 103-120.

[8] O. N. Ricceri, "Classical solutions of the problem $\dot{x} \in F(t, x, \dot{x}), x\left(t_{0}\right)=x_{0}, \dot{x}\left(t_{0}\right)=y_{0}$ in Banach spaces", Funkcialaj Ekvacioj, 34 (1991), 127-141.

Department of Mathematics, Bharathiar University Coimbatore-641 046, Tamil Nadu, India.

* Gobi Arts College, Gobichettipalayam-638 453, Tamil Nadu, India. 